\title{
El arbitraje internacional como un sistema transnacional de justicia
}

\author{
Emmanuel Gaillard
}

En el presente artículo, Emmanuel Gaillard desarrolla ampliamente las tres principales visiones que existen en el arbitraje internacional: la corriente monolocalista, la Westfaliana y la trasnacional. Asimismo, desarrolla sus consecuencias prácticas y el rol de la Convención de Nueva York de 1958 en el reconocimiento del arbitraje como un sistema trasnacional de justicia.

Profesor de Derecho en Sciences Po Law School; Jefe del Grupo Internacional de Arbitraje de Shearman \& Sterling LLP; Miembro de la ICCA; "Star-individual" del Ránking Arbitration (International) - Global-wide.

El presente artículo fue publicado en inglés en Albert Jan van den Berg (ed.) Arbitration: The Next Fifty Years ICCA Congress Series 16, Geneve 2011, Kluwer Law International, pp. 66-73. La traducción de este artículo ha estado a cargo de la Comisión de Edición de Forseti. Cualquier error es de exclusiva responsabilidad de los traductores. 


\section{El arbitraje internacional como un sistema transnacional de justicia}

\section{INTRODUCCIÓN}

Cada vez más se reconoce al arbitraje internacional como un sistema transnacional de justicia, y si no es como un orden jurídico autónomo, algunas veces es considerado como un orden jurídico arbitral. ${ }^{1}$ No obstante, esta evolución continúa generando robustas controversias sobre el alcance de la autonomía del arbitraje internacional de los ordenamientos jurídicos nacionales; y el rol, si alguno, que debería tener la sede de arbitraje sobre el proceso arbitral.

Este debate generalizado sugiere que, hasta cierto punto, todavía se encuentra mal definida la duradera relación entre la autonomía y el arbitraje internacional. También demuestra que lo que se entiende por autonomía, sin más, no permite dar respuesta a algunas preguntas más fundamentales y prácticas en el arbitraje internacional, como por ejemplo, las preguntas relacionadas al derecho aplicable, al procedimiento y a la ejecución de laudos.

Un enfoque más fundamental se basa en examinar las razones de la fuerte divergencia de opiniones entre aquellos que califican al arbitraje internacional en un sistema legal nacional y aquellos que reconocen el carácter transnacional del proceso. Estas diferencias no pueden solamente ser explicadas por razones de autonomía, o por enfocar aspectos prácticos aislados del arbitraje internacional. En su lugar, uno debe encontrar la fuente (o fuentes) de la validez y legitimidad del proceso arbitral y del subsecuente laudo arbitral. En este sentido, tres formas de ver el arbitraje internacional parecen ser una línea divisoria, cada una con una base teórica distinta y

1 Para una discusión sobre la evolución y uso de la expresión "orden jurídico arbitral", ver Gaillard, Emmanuel, Legal Theory of International Arbitration, Martinus Nijhoff Publishers, 2010, pp. 38-46. Sobre el reconocimiento en ciertas jurisdicciones de la existencia de una orden jurídico arbitral, ver Hasher, Dominique, "The Review of Arbitral Awards by Domestic Courts - France". En: Gaillard, Emmanuel (ed.), IAI Series on International Arbitration, No. 6. The Review of International Arbitral Awards, Juris, 2010, p. 97 ("The French concept of arbitration is based on the premise that there is an arbitral legal order, which is distinct from the legal order of individual States (...) It is this arbitral legal order - and no national legal order - that confers juridicity to arbitration."); Ancel, Jean-Pierre, "The Decree of 13 January 2011: Increasing the Efficiency of Arbitration in France", The 13 January 2011 Decree, The New French Arbitration Law, Paris the Home of International Arbitration, 2011,p. 9 (noting that the French view of international arbitration law is based on "the recognition of the existence of a truly autonomous arbitral legal order"); Draetta, Ugo, Reporte dictado en el Seminario Journée d'étude en I'honneur de Giorgio Shiavoni : La résolution des différends commerciaux en Méditerranée : quel rôle pour l'arbitrage?: "L'arbitrage international est-il une fonction publique?", Camera Arbitrale di Milano, 27 de junio de 2011. 
que conllevan a extremas consecuencias prácticas. Estas visiones serán descritas más abajo (II), antes de darle mayor ponderación al rol de la Convención de Nueva York de $1958^{2}$ en este debate (III).

\section{LAS REPRESENTACIONES DEL ARBITRAJE INTERNACIONAL}

Existen tres visiones distintas sobre el arbitraje internacional, definidas por lo que sus defensores consideran ser la fuente de legitimidad del fenómeno: la visión monolocalista, la visión Westfaliana y la visión transnacional. ${ }^{3}$ Estas visiones, construcciones mentales o representaciones ${ }^{4}$, están fuertemente influenciadas en los puntos de vista a preguntas que van desde si los árbitros tienen competencia para determinar su propia competencia, el desarrollo del procedimiento arbitral, la determinación del derecho aplicable al fondo de controversia, o el destino del laudo arbitral, incluyendo la siempre controversial pregunta de si un laudo anulado podrá ser ejecutado en otras jurisdicciones.

Este artículo se enfocará en desvanecer dos comunes malentendidos que se encuentran en el corazón de los fundamentos filosóficos de las representaciones del arbitraje internacional y sus consecuencias prácticas: Primero, ninguna de estas representaciones sugiere que el arbitraje internacional no deriva su legitimidad o validez finalmente de los Estados (1). Segundo, si es que uno es o no consciente de su existencia, estas representaciones acarrean consecuencias prácticas de importancia para todos los jugadores en el campo del arbitraje internacional, no solamente para los árbitros. (2)

\section{La fuente de la legitimidad y validez del arbitraje internacional}

Las tres representaciones del arbitraje internacional están definidas por aquello que se considera ser la fuente de legitimidad del arbitraje internacional. Principalmente, cuál es la fuente de poder que tienen los árbitros de resolver la controversia. Este supuesto

2 Convención sobre el Reconocimiento y Ejecución de Laudos Arbitrales Extranjeros, hecho en Nueva York el 10 de junio de 1958 (en adelante, la Convención de Nueva York o la Convención). Al 30 de septiembre de 2011, 146 Estados eran partes de la Convención de Nueva York. La situación actual de los países miembros de la Convención de Nueva York puede ser visto en:

http://www.uncitral.org/uncitral/es/uncitral_texts/arbitration/NYConvention_status.html

3 Ver: Gaillard, Emmanuel, Legal Theory of International Arbitration, Martinus Nijhoff Publishers, 2010, pp. 38-46.

Ver también: Gaillard, Emmanuel, "Three Philosophies of International Arbitration". En Rovine, Arthur W. (ed.), Contemporary Issues in International Arbitration and Mediation: The Fordham Papers 2009, Martinus Nijhoff Publishers, 2010.

4 Para un análisis de la noción de representación desde una perspectiva de la ciencia cognitivia, ver Gaillard, Emmanuel, "The Representations of International Arbitration". En: Journal of International Dispute Settlement, 2010, pp. 1-11. 
tiene implicaciones de largo alcance sobre cómo uno ve la totalidad del proceso arbitral.

La primera y más tradicional representación es la visión monolocalizadora, que considera el derecho de la sede del arbitraje como la única fuente de legitimidad y validez del arbitraje internacional, o como algunos defensores de esta visión dirían, el "país de origen". En esta visión, la sede del arbitraje es más que un lugar elegido por conveniencia o neutralidad. La sede provee la base exclusiva de la naturaleza vinculante del arbitraje. En consecuencia con esta visión, si el lugar del arbitraje es México, es un arbitraje mexicano. Incluso si la disputa es entre una compañía francesa y una compañía americana y que se rige por el derecho sueco. Si a estas diversas partes se les permite arbitrar, es porque el ordenamiento jurídico mexicano les ha preautorizado la posibilidad de hacerlo. El laudo que resulte de ese arbitraje es considerado un laudo mexicano.

La segunda representación es la visión Westfaliana. Esta considera que el arbitraje internacional es un proceso legítimo porque existen un número de Estados que están preparados para reconocer la legitimidad de un proceso basado en la común intención de las partes, así como también en la naturaleza vinculante del laudo arbitral. Esta visión es una desviación radical de la visión monolocalizadora en dos aspectos. Primero, el proceso arbitral ya no se encuentra pre-autorizado, sino que se encuentra legitimado a posteriori si es que el laudo cumple con los criterios de ejecución del Estado. Segundo, esta visión considera que el arbitraje internacional puede derivar su legitimidad de una pluralidad de sistemas legales, incluyendo el Estado o Estados en los que se ejecutará. Bajo esta representación, el derecho de la sede es solamente uno entre otros sistemas legales potencialmente relevantes. $Y$ la sede es considerada más como un lugar físico donde se llevara a cabo el arbitraje, que un foro legal que definirá el alcance del rol del árbitro, del mismo modo como haría para una corte local. Esta visión de pluralidad judicial es mejor descrita como Westfaliana porque está basada en un modelo en el que cada Estado tiene un título legítimo para decidir por sí mismo las condiciones bajo las que considerara válido el proceso arbitral y el laudo arbitral como merecedor de ejecución. ${ }^{5}$

La tercera representación, la visión transnacional, toma un paso más que la aproximación Westfaliana al contemplar a los Estados de manera colectiva, en vez de solamente de manera individual. Esta representación reconoce la fuente de la legitimidad del arbitraje como arraigada en las visiones desarrolladas colectivamente

5 Para un ejemplo de esta aproximación, Arthur von Mehren, quien describió elocuentemente en una conferencia en Tel Aviv en 1986 la naturaleza ambulatoria del arbitraje y el hecho de que, a diferencia de un juez, los árbitros no tienen una lex fori: Von Mehren, Arthur T., "Limitations on Party Choice and the Governing Law: Do They Exist for International Commercial Arbitration?", The Mortimer and Raymond Sackler Institute of Advanced Studies, Tel Aviv University, 1986. 
por la comunidad internacional por medio de instrumentos como la Convención de Nueva York de 1958, la Ley Modelo Uncitral y numerosas directrices que expresan una visión común sobre cómo el arbitraje debe ser conducido para que sea reconocido como un medio legítimo de solución de controversias. En otras palabras, en esta visión, la fuente de validez y legitimidad del proceso arbitral se encuentra en la actividad normativa colectiva de los Estados. Esta representación también corresponde a la fuerte percepción de los árbitros de que ellos no administran justicia de parte de ningún Estado en particular, sino que ellos juegan un rol judicial para el beneficio de la comunidad internacional.

Algunos han equivocado esta representación transnacional al ser una visión de anarquía judicial. Michael Reisman ha sugerido que "los defensores del arbitraje suelen asumir (...) que el arbitraje es un procedimiento conceptualmente y políticamente bastante independiente del aparato del Estado". Continúa describiendo a estos defensores como "aquellos que desean pensar en el arbitraje sin ningún rol para el Gobierno". Esta posición confunde la noción de un orden legal autónomo con un orden legal a-nacional, que sería caracterizado como un rechazo o una oposición a sistemas legales nacionales. ${ }^{6}$ En contraste, la representación transnacional considera que el arbitraje internacional está anclado en la colectividad de sistemas legales. Como tal, esta visión de colectivismo judicial acoge, mas no rechaza, las leyes derivadas de sistemas legales nacionales.

Por lo tanto, ninguna de estas tres representaciones anteriormente descritas sugiere que el arbitraje internacional promueve un sistema internacional de justicia que esta "flotando en el cielo transnacional", ${ }^{7}$ completamente divorciado de los sistemas legales nacionales de los Estados. Al contrario, la pregunta relevante para entender estas tres visiones es qué Estado, o Estados, proveen la fuente relevante suficiente de legitimidad y validez para el acuerdo arbitral, el proceso arbitral y el resultante laudo arbitral.

$6 \quad$ En contraste a la representación transnacional del arbitraje internacional, la doctrina de la lex mercatoria surgió de una visión más crítica sobre los sistemas legales nacionales y de la deficiencia percibida de aquellos sistemas de tomar en cuenta disputas que surgían en un entorno comercial mundial y por medio de su selectividad, constituían una forma de "Darwinismo legal". Ver: Loquin, Eric, "Où en est la lex mercatoria?". En: Souveraineté Étatique et Marchés Internationaux à la Fin du 20ème Siècle. A Propos de 30 Ans de Recherches du CREDIMI. Mélanges en I'Honneur de Philippe Kahn, Litec, 2000, p. 26; Loquin, Eric, "Les règles matérielles internationales". En: Collected Courses of the Hague Academy, vol. 322, 2006, Sect. 503; para una discusión de esta idea, ver: Gaillard, Emmanuel, Legal Theory of International Arbitration, Martinus Nijhoff Publishers, 2010, pp. 46-47.

7 Otro ejemplo de esta percepción erronea de una filosofía transnacional es Bank Mellat c. Helliniki Techniki SA, donde Kerr $U$ señaló que: "[d]espite suggestions to the contrary by some learned writers under other systems, our jurisprudence does not recognise the concept of arbitral procedures floating in the transnational firmament, unconnected with any municipal system of law" [1984] 1 QB 291, 301. 


\section{Consecuencias prácticas de las representaciones}

Estas visiones sobre el arbitraje internacional que compiten entre sí, no solamente nutren un debate teórico, sino que se trasladan en consecuencias bastante prácticas. Estas influencian no solo cómo los árbitros abordan varios aspectos en un arbitraje internacional, sino que también a la aproximación tomada por todos los otros jugadores en el campo, incluyendo las partes, los abogados y jueces nacionales.

Por supuesto, las representaciones tienen consecuencias significativas para los árbitros y para los arbitrajes que ellos presiden. Por ejemplo, al elegir el Derecho aplicable, ${ }^{8}$ un árbitro que se adhiere a la primera representación y se encuentra en México, considerará se encuentra obligado por las normas de conflicto de leyes del derecho mexicano. Un árbitro que adopta la visión westfaliana no tendría problemas en elegir, entre las varias reglas de conflicto de Derecho en juego, la regla de elección del derecho que considera apropiada al arbitraje en juego. Un árbitro que se adhiere a la representación transnacional será más propenso a mirar las tendencias internacionales en la determinación de normas de conflicto de leyes o, en casos apropiados, la regla que refleja el consenso de las naciones en resolver un tema sustancial particular. Como el árbitro westfaliano, este árbitro transnacional no se considerará a sí mismo obligado por las normas de conflicto de leyes mexicanas simplemente porque él o ella se encuentra en México. No obstante, a diferencia del árbitro westfaliano que se ve a sí mismo como titular de autonomía ilimitada, un árbitro que adopta la visión transnacional aprecia las tendencias desarrolladas por la comunidad internacional para que lo guíe. Mientras que la visión westfaliana está caracterizada por un margen de imprevisibilidad, la visión transnacional apunta a promover certeza a través de apoyarse en principios mayoritarios y rechazar idiosincrásicas o anticuadas reglas de Derecho.

Este ejemplo de la elección del derecho aplicable al fondo de la controversia ilustra los efectos de largo alcance que las representaciones pueden tener en los árbitros y cómo ellos a la vez tienen un impacto significativo en el resultado del arbitraje mismo. Lo mismo es verdad para todos los otros jugadores en el campo del arbitraje internacional.

Para tomar otro ejemplo, estas representaciones influyen en gran manera a las Cortes nacionales. Por ejemplo, cuando a una Corte se le pide que ejecute un laudo arbitral que fue anulado en el país del lugar del arbitraje. Las cortes nacionales que adoptan una visión monolocal considerarán un laudo otorgado en México como un laudo

$8 \quad$ Para una discusión detallada de las consecuencias de las representaciones del arbitraje internacional en el procedimiento aplicable y las reglas de Derecho, ver: Gaillard, Emmanuel, Legal Theory of International Arbitration, Martinus Nijhoff Publishers, 2010, pp. 93-134. 
mexicano, incluso si ninguna de las partes proviene de México y lo tratarán como si fuera un fallo local otorgado por una corte mexicana. Consecuentemente, si el laudo es anulado en México, no hay nada que reconocer en otro Estado.

Las cortes con una visión westfaliana no se adherirán necesariamente a la forma en que un laudo emitido en México es tratado por el ordenamiento jurídico mexicano. Las cortes no considerarían la determinación de una corte mexicana de que un laudo emitido en México no es válido; por ejemplo, por un error de los árbitros de cumplir con una regla procedimental local, como si tuviera un efecto legal territorial absoluto. Las cortes de los demás países considerarían que son libres de hacer sus propias determinaciones sobre si el error debería conllevar la anulación del laudo como un instrumento legal vinculante. Por lo tanto, las cortes inglesas podrían ejecutar un laudo arbitral que las cortes mexicanas han anulado.

Las cortes que se adhieren a la tercera visión de representación transnacional aceptan el mismo resultado pero por diferentes razones. Ellas buscarán qué se considera como un laudo válido por los estándares internacionales, como aquellos establecidos en la Convención de Nueva York. Su adherencia a estos estándares internacionales no sugiere que estas Cortes no ejercitan ningún control sobre el reconocimiento del laudo en su propio ordenamiento jurídico. Por lo contrario, la Convención de Nueva York ha preservado la discreción de todo ordenamiento jurídico de decidir, por sí mismo, si es que un laudo cumple o no con las condiciones de reconocimiento y ejecución. ${ }^{9}$ En este sentido, los ordenamientos jurídicos nacionales continúan dándole forma al arbitraje internacional tanto por su adherencia a estándares colectivos como desarrollando jurisprudencia nacional sobre arbitraje cuando ejercitan su discreción dentro de esos estándares.

\section{EL ROL DE LA CONVENCIÓN DE NUEVA YORK DE 1958}

Debido a su rol crucial en el desarrollo del arbitraje internacional durante los últimos cincuenta años, la Convención de Nueva York de 1958 provee una útil muestra de la pregunta de si es que el arbitraje internacional puede ser visto como un sistema transnacional de justicia.

Las reglas establecidas por la Convención de Nueva York representan un movimiento colectivo de pro-ejecución de parte de la comunidad internacional. A pesar de la aproximación relativamente directa de la Convención sobre la promoción de la

$9 \quad$ Desde un punto de vista procedimental, el Art. III de la Convención de Nueva York establece que "[e]ach Contracting State shall recognize arbitral awards as binding and enforce them in accordance with the rules of procedure of the territory where the award is relied upon")." (énfasis agregado). En otras palabras, los estándares de acuerdo con los cuales un laudo "será" (shall) reconocido son aquellos del Estado donde el reconocimiento y ejecución sea invocado. 
ejecución al limitar los motivos de revisión para una ejecución de laudo arbitral, esta ha sido interpretada por algunos como creador de una jurisdicción jerárquica entre ordenamientos jurídicos nacionales que revisan laudos. Esta interpretación no solo no tiene fundamento en el texto de la Convención, sino que también es contraria a los objetivos fundamentales de la Convención.

Un defensor de esta idea, Michael Reisman, quien ha argumentado que la Convención de Nueva York crea una "arquitectura normativa" de arbitraje internacional, que otorga competencia a "dos niveles de competencia de revisión, haciendo una distinción entre las llamadas jurisdicciones 'primarias' o del 'lugar' y jurisdicciones 'secundarias' o 'de ejecución'". ${ }^{10}$ Esta visión sugiere que la Convención establece, por un lado, una jurisdicción principal, aquella de la del lugar del arbitraje - sin importar los otros criterios adoptados por la Convención sobre el derecho elegido por la partes para que rija el arbitraje $-y$, por otro lado, una jurisdicción secundaria (o jurisdicciones secundarias) en el lugar (o lugares) de ejecución.

En realidad, el objetivo de la Convención de Nueva York es mucho más sencillo. Es facilitar el reconocimiento y ejecución de laudos arbitrales, no para otorgar competencia relativa a ordenamientos jurídicos nacionales. La idea de que la Convención de Nueva York pondría al lugar del arbitraje en el tope de una jerarquía jurisdiccional para finalidades de ejecución es contraria a sus objetivos fundamentales. ${ }^{11}$ Si la interpretación es aceptada, cambiaría el enfoque del laudo mismo, que es la materia objeto de la Convención, y del proceso judicial que vincula al laudo en el país donde fue dictado, e iría en contra de uno de los mayores logros de la Convención de Nueva York. En efecto, hay que recordar que los redactores de la Convención buscaban abolir el requerimiento del doble exequátur, que regía la ejecución bajo la Convención de Ginebra sobre la Ejecución y Reconocimiento de Laudos Arbitrales Extranjeros de 1927. Hasta 1958, los laudos tenían que ser aprobados por las cortes de la sede del arbitraje antes de que pudieran ser ejecutados en otro lugar. Por lo que la Convención de Geneva de 1927 reconoció el "país de origen" como poseedor de competencia principal en determinar si un laudo era válido. En ese momento, este pudo organizar al mundo en una jurisdicción primaria, aquella del sitio, y una jurisdicción secundaria. Los redactores de la Convención de Nueva York quisieron precisamente alejarse de este sistema anti-ejecutorio y adoptar un sistema en el que la revisión de laudos para su ejecución sea limitada a motivos definidos en la

10 Reisman, W. Michael y Richardson, Brian, "Tribunals and Courts: An Interpretation of the Architecture of International Commercial Arbitration". En: Albert Jan van den Berg (ed.), ICCA Congress Series 16, Geneve 2011, Kluwer Law International, p. 25.

11 Es también contrario a la letra de la Convención, que pone a la sede y al derecho bajo el que el laudo será otorgado, en el mismo nivel. Ver el artículo V(1)(e) de la Convención. Por lo tanto, si la Convención fuera a ser vista como si estuviese asignando jurisdicciones entre países, lo cual no hace, habría creado jurisdicciones primarias (plural), no una jurisdicción primaria. 
Convención. ${ }^{12}$ La abolición del doble exequátur ha sido masivamente aplaudida desde 1958 como un gran paso a un sistema pro-ejecución. El hecho de que los redactores de la Convención se alejaron de la noción de que la sede del arbitraje es de alguna manera el país controlante, no sugiere que ningún Estado tenga control sobre el proceso arbitral; más bien, ellos cambiaron la autoridad de revisión de laudos para ejecución a los Estados al lugar (o lugares) de ejecución.

Por esta razón, la Convención no hace mención alguna sobre el alcance de la revisión de laudos arbitrales para efectos de anulación en el sitio del arbitraje. No dice, por ejemplo, que la sede debe tener un máximo de cinco, seis, o siete motivos para anular. No existe una violación de la Convención si, por ejemplo, las Cortes en los Estados Unidos revisan un laudo por "desconocimiento manifiesto de la ley" con ocasión de anular un laudo otorgado en los Estados Unidos. Si, por otro lado, un Estado fuese a revisar el "desconocimiento manifiesto de la ley" de un árbitro con fines de ejecutar un laudo que se encuentre dentro de los ámbitos de la Convención, estaría en violación de la Convención.

Por el contrario, la Convención no previene al Estado de la ejecución de ser más permisivo sobre los motivos establecidos en la Convención que representan el estándar máximo de revisión aceptable bajo la Convención cuando a un país se le pide que reconozca y ejecute un laudo arbitral. ${ }^{13}$ Si un Estado revisa los méritos de la disputa, viola la Convención. Si un Estado utiliza un motivo diferente que aquellos estipulados en la Convención para denegar la ejecución, viola la Convención. Pero si un Estado decide ejecutar laudos de acuerdo a estándares más liberales, está libre para hacerlo. Más específicamente, la Convención no prohíbe a un Estado de ejecutar un laudo que ha sido anulado en la sede del arbitraje o en el país del cual surge la ley que gobierna

12 Ver por ejemplo Art. VII (2) de la Convención de Nueva York que establece que "El Protocolo de Ginebra de 1923 relativo a las cláusulas de arbitraje y la Convención de Ginebra de 1927 sobre la ejecución de las Sentencias Arbitrales Extranjeras dejarán de surtir efectos entre los Estados Contratantes a partir del momento y en la medida en que la presente Convención tenga fuerza obligatoria para ellos".

Este hecho se aclarece por la más favorable provisión de la Convención de Nueva York que expresamente provee que las Cortes deben prestar atención a las leyes domesticas que sean más favorables que la Convención: "1. Las disposiciones de la presente Convención no afectarán la validez de los acuerdos multilaterales o bilaterales relativos al reconocimiento y la ejecución de las sentencias arbitrales concertados por los Estados Contratantes ni privarán a ninguna de las partes interesadas de cualquier derecho que pudiera tener a hacer valer una sentencia arbitral en la forma y medida admitidas por la legislación o los tratados del país donde dicha sentencia se invoque." Un número de Cortes domesticas han ejercido esta discreción al aplicar provisiones más favorables de Derecho doméstico. Ver: Gaillard, Emmanuel, "The Relationship of the New York Convention with Other Treaties and with Domestic Law". En: Gaillard, Emmanuel y Di Pietro, Domenico (eds.) Enforcement of Arbitration Agreements and International Arbitral Awards, Camerún, Mayo, 2008, en pp. 70-71 y 76-86. 
el arbitraje. ${ }^{14}$ Por lo tanto, cualquier posición tomada por las cortes de la sede del arbitraje con respecto a la validez del laudo arbitral no tiene un efecto vinculante en otros ordenamientos jurídicos.

La aceptación generalizada de la Convención de Nueva York por los Estados evidencia un alejamiento del paradigma tradicional que otorga una discreción injustificada a la sede del arbitraje. Al darle algo de discreción a los ordenamientos jurídicos nacionales, al lugar (o lugares) de ejecución, de revisar laudos arbitrales, la Convención parece, por lo menos, acomodar la representación westfaliana del arbitraje internacional. Pero la Convención es primordialmente una declaración de los estándares globales dominantes a los que Estados contratantes aceptan adherirse, estableciendo las fronteras dentro de las que ellos tendrán que ejercer su discreción. De esta manera, la Convención ejemplifica la actividad normativa y colectiva de los Estados en los que la legitimidad y validez del orden jurídico arbitral trasnacional se encuentra anclado.

14 Para un análisis más detallado sobre la ejecución de laudos arbitrales anulados en el sitio en relación a las tres representaciones del arbitraje internacional, ver: Gaillard, Emanuel, "The Representations of International Arbitration". En: New York Law Journal, 4 de octubre de 2007. 